\title{
Quand l'urine cisaille les cellules rénales
}

$>$ Le cisaillement - ou shear stress - est une contrainte mécanique dont le rôle en physiopathologie vasculaire n'est plus à démontrer. Toutefois, le cisaillement apparaît maintenant aussi comme un mécanisme essentiel pour la fonction rénale et de plus en plus d'arguments indiquent même qu'il contribuerait au développement des néphropathies. Le but de cette revue est de proposer un état de l'art sur les rôles physiologiques du shear stress urinaire et ses implications en pathologie. <

Le cisaillement urinaire : une histoire d'écoulement

Le cisaillement - ou fluid shear stress (FSS) en anglais est la force qu'exerce le frottement d'un fluide en mouvement sur la paroi d'un tube. II dépend du débit du fluide, de sa viscosité ainsi que du diamètre du tube. C'est au niveau vasculaire que le cisaillement est le mieux caractérisé. Généré par la circulation du sang, le FSS vasculaire s'élève à environ $2 \mathrm{~Pa}$ dans les artères et $0,4 \mathrm{~Pa}$ dans les veines. II induit des modifications fonctionnelles et structurales des vaisseaux et il est très fortement associé à la localisation des plaques d'athérosclérose [1]. Au niveau rénal, on peut également mettre en évidence la présence d'un cisaillement. Chaque rein est en effet composé d'un million de petits tubes, les néphrons, dans lesquels s'écoule l'urine en formation (Figure 1). Les cellules qui bordent la paroi des néphrons sont donc soumises en permanence à un cisaillement, créé cette fois-ci par le déplacement de l'urine (Figure 1). Plus faible que dans les vaisseaux, le FSS urinaire est de l'ordre de 0,1 Pa à l'entrée du tubule proximal [2,3], là où se déverse l'urine primitive. À l'heure actuelle, peu de travaux ont étudié les effets du cisaillement urinaire. Néanmoins, les quelques résultats publiés sur ce sujet montrent que les cellules tubulaires rénales, au même titre que les cellules endothéliales des vaisseaux, sont capables de répondre au cisaillement. Mieux - ou pis - encore, certains résultats suggèrent que le shear stress urinaire pourrait être impliqué dans le développement des néphropathies.

Vignette (Photo @ Inserm - Éric Dehausse).

\section{Un nouveau rôle pour}

le shear stress dans

les néphropathies
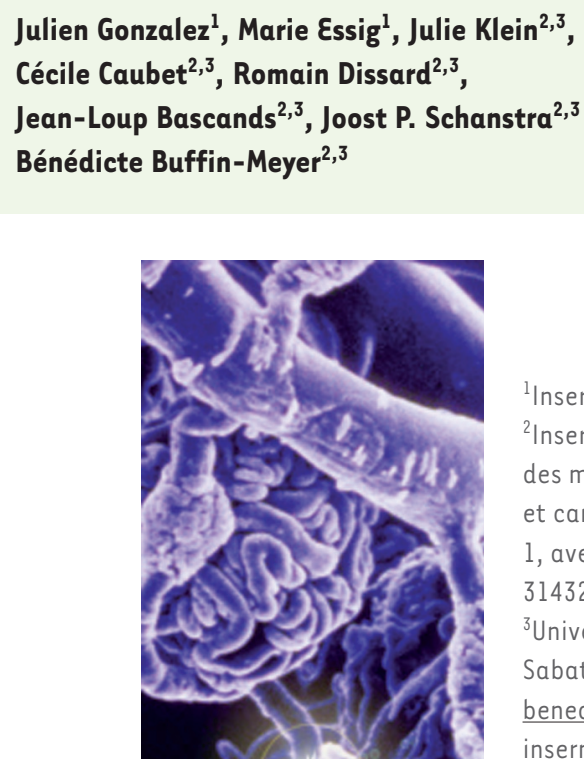

${ }^{1}$ Inserm U850, Limoges, France ;

'Inserm U1048, institut des maladies métaboliques et cardiovasculaires (I2MC), 1 , avenue Jean Poulhès, 31432 Toulouse Cedex 4, France ; ${ }^{3}$ Université Toulouse III PaulSabatier, Toulouse, France. benedicte.buffin-meyer@ inserm.fr

La perception du cisaillement est importante pour les cellules tubulaires

Contrôle des fonctions glomérulaires et tubulaires

On sait depuis longtemps que les variations du débit d'écoulement du fluide urinaire dans le tubule rénal modifient les échanges tubulaires tels que les réabsorptions d'eau, de $\mathrm{NaCl}$ et de bicarbonates ou la sécrétion de $\mathrm{K}^{+}$. Elles sont aussi à l'origine du rétrocontrôle tubuloglomérulaire, mécanisme par lequel les cellules de la macula densa modifient les résistances artériolaires glomérulaires pour contrôler la filtration glomérulaire. Toutefois, la majorité de ces travaux ont utilisé des segments de néphrons microperfusés. Or, une variation du débit du fluide dans ces modèles modifie non seulement le FSS urinaire mais également au moins deux autres paramètres : la pression exercée à la surface des cellules et le degré d'étirement de la membrane cellulaire. $\varepsilon n$ utilisant des dispositifs pour s'affranchir des paramètres de pression et d'étirement, des études ont récemment analysé les effets du cisaillement sur les propriétés de transport des cellules rénales. Ainsi, l'exposition des cellules tubulaires proximales au FSS stimule l'expression des protéines de transport $\mathrm{NHE3}$ (échangeur $\mathrm{Na}^{+} / \mathrm{H}^{+}$ apical), $\mathrm{H}^{+}$-ATPase et $\mathrm{Na}^{+} / \mathrm{K}^{+}$-ATPase ainsi que leur adressage à la membrane plasmique [4]. Dans les cellules du canal collecteur, le FSS induit la translocation des aquaporines AQP2 à la membrane apicale [5] et favorise l'expulsion de $\mathrm{K}^{+}$via des canaux potassiques 

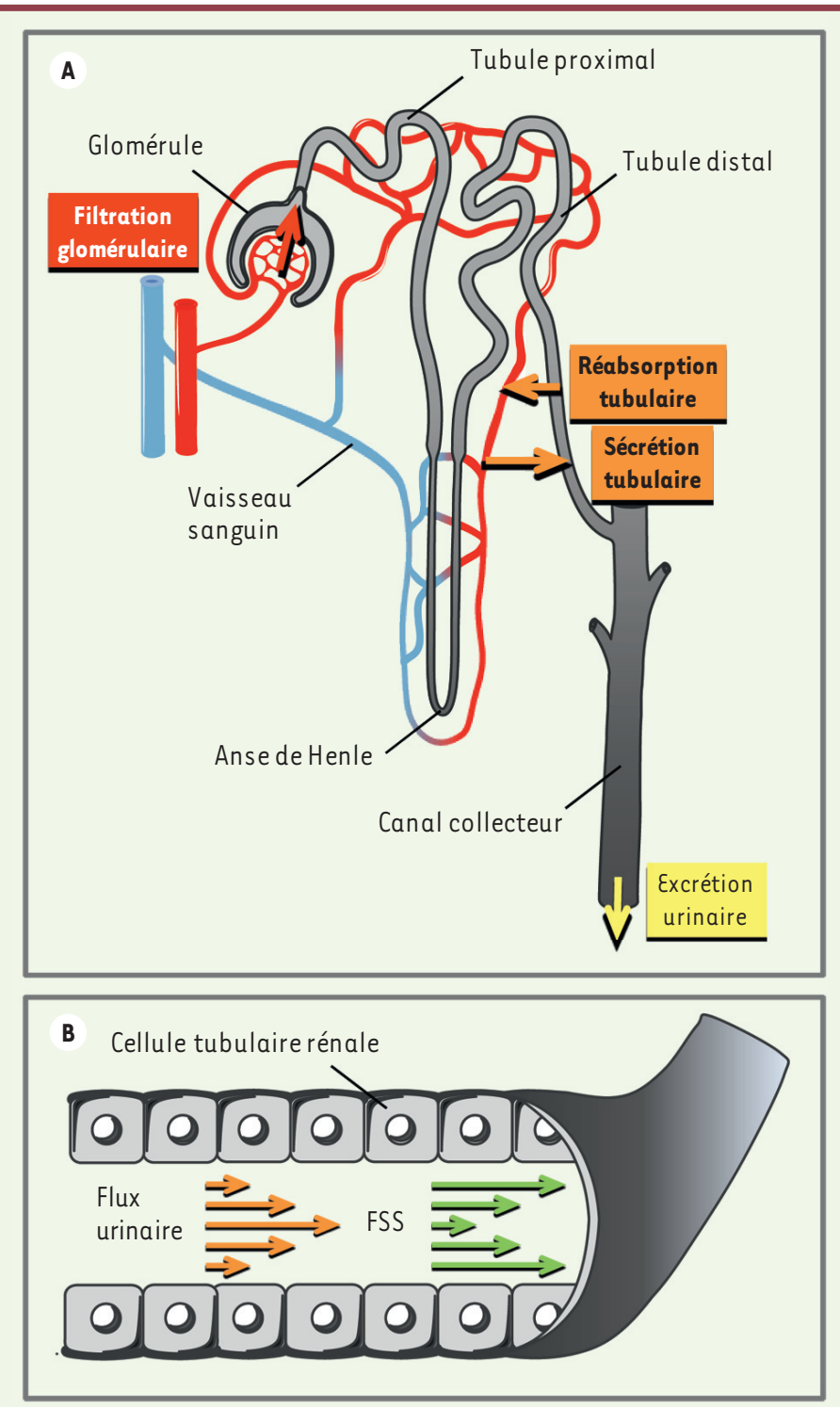

Figure 1. Le cisaillement urinaire est induit par l'écoulement de l'urine. Les reins ont un double rôle : ils débarrassent le sang de ses déchets et ils veillent à ce que la quantité d'eau et d'ions de notre corps soit optimale. Comment? En fabriquant l'urine grâce à ses néphrons. $\boldsymbol{A}$. Le glomérule produit d'abord une urine primitive par filtration du plasma. Le tubule rénal modifie ensuite la composition de cette urine primitive par le biais d'échanges (réabsorption ou sécrétion) avec le sang. Le tubule rénal est organisé en quatre segments successifs : le tubule proximal, l'anse de Henle, le tubule distal et le canal collecteur, chacun exprimant des fonctions différentes (transport, métabolisme, régulation). Une fois synthétisée, l'urine est ensuite excrétée hors des néphrons, puis hors de l'organisme. B. Au fur et à mesure qu'elle s'écoule dans la lumière tubulaire, l'urine en formation génère une force de friction à la surface des cellules tubulaires : c'est ce qu'on appelle le cisaillement urinaire. Son intensité (flèche verte) est faible au centre du tubule, là où la vitesse de l'urine (flèche orange) est maximale. En revanche, contre la paroi tubulaire, le cisaillement est élevé et la vitesse d'écoulement de l'urine est alors minimale. sensibles au calcium [6]. II y entraîne également la libération du monoxyde d'azote et des prostaglandines દ2 [7-9], deux modulateurs des échanges tubulaires. Enfin, des études utilisant des ovocytes montrent que le FSS augmente la probabilité d'ouverture du canal ENac, protéine qui assure la réabsorption de $\mathrm{Na}^{+}$dans le canal collecteur [10]. Ces résultats suggèrent donc que le cisaillement urinaire participe, en tant que tel, au contrôle des échanges tubulaires.

\section{Réarrangement du cytosquelette cellulaire}

Des cellules proximales en culture, maintenues en conditions statiques, présentent de nombreuses fibres de stress d'actine réparties dans le cytosol. En revanche, en réponse au shear stress, ces fibres cytosoliques disparaissent et on observe un renforcement du réseau d'actine au pôle apical et en position latérale, associée à un assemblage des jonctions intercellulaires serrées et d'ancrage [11-13]. Cette réorganisation du cytosquelette d'actine joue un rôle important dans les effets du shear stress puisque sa perturbation par la cytochalasine $D$ abolit l'activation des protéines de transport $\mathrm{NH} \varepsilon 3$ et $\mathrm{Na}^{+} / \mathrm{K}^{+}$-ATPase induite par le FSS, et réduit la réabsorption de fluide induite par l'augmentation de la perfusion tubulaire $[4,14,15]$. De manière surprenante cependant, ce remaniement diffère de celui qui s'opère dans les vaisseaux sanguins puisque les cellules endothéliales répondent au shear stress en rompant l'actine périphérique et en formant des fibres de stress qui s'alignent en direction du flux [11].

Le réseau de microtubules contribue également aux effets du FSS rénal : les drogues qui modifient sa dynamique diminuent certaines réponses au FSS, notamment l'activation de la $\mathrm{H}^{+}$-ATPase $[4,16]$.

\section{Différenciation cellulaire}

Le shear stress pourrait favoriser la différenciation in vitro des cellules tubulaires et/ou le maintien in vivo d'un phénotype différencié. En effet, si l'on compare l'organisation du cytosquelette d'actine et des jonctions intercellulaires dans les cellules tubulaires proximales cultivées in vitro et dans le tubule proximal in vivo, il s'avère que les cellules exposées in vitro au FSS présentent une organisation plus proche des conditions physiologiques in vivo que celles maintenues en conditions statiques [11-13] (Encadré 1). D'autre part, les cellules MDCK (représentatives du canal collecteur) cultivées à confluence s'organisent en monocouches et forment spontanément des dômes. Ces dômes correspondent à des cellules détachées de leur support d'adhésion, mais liées les unes aux autres par leurs domaines latéraux. Ils résultent de l'accumulation de 


\section{Un point de méthodologie}

Notre corps est sujet à de multiples écoulements de fluides. Or, dès lors qu'un liquide se déplace sur une surface, il expose cette surface à un cisaillement. Pour cette raison, de nombreuses cellules de l'organisme, en plus des cellules endothéliales (écoulement du sang) ou rénales (écoulement de l'urine), subissent un cisaillement (tableau ci-dessous). L'exposition de différents types cellulaires à un cisaillement influence la morphologie, l'expression des gènes/protéines et l'activité biologique des cellules. De plus, le réseau d'actine est mieux structuré dans les cellules (tubulaires rénales) cisaillées que dans celles maintenues en conditions statiques. Pour le cas particulier des thématiques portant sur des tissus soumis in vivo à un cisaillement, on peut donc légitimement se demander s'il est pertinent de travailler in vitro avec des cellules cultivées dans un milieu statique. Ne faudrait-il pas plutôt appliquer en permanence un cisaillement physiologique à la surface de ces cellules ? La question reste posée...

Exemples de tissus - autres que les vaisseaux ou les néphrons - soumis in vivo à un cisaillement

\section{Tissu}

Paroi des voies urinaires

Uretères/vessie/urètre

Paroi du tube digestif

Esophage

Cryptes gastriques

Entérocytes intestinaux

\section{Glandes exocrines}

Glandes mammaires

Glandes salivaires

Foie/vésicule biliaire

Pancréas exocrine

Vésicules séminales/prostate

Glandes lacrymales

\section{Voies respiratoires}

Voies aériennes / alvéoles

\section{Autres}

Os/cartilage

Muscle

Neurones

\section{Fluide en mouvement}

Urine

Bol alimentaire
Chyme
Chyle

Canaux excréteurs associés

Canaux galactophores

Lait

Canaux annexés à la cavité buccale Salive

Canaux hépatiques

Bile

Suc pancréatique

Canal pancréatique

Canal éjaculateur/urètre

Canal lacrymal

Larmes

Air

Liquide interstitiel

Liquide interstitiel

Liquide céphalorachidien

liquide entre la base de la monocouche et le support, suite au transport de fluide du pôle apical vers le pôle basal de la couche cellulaire. II a récemment été observé que l'application d'un shear stress sur des cellules MDCK entraîne la disparition des dômes [17]. L'hypothèse formulée par les auteurs pour expliquer cette observation est que le cisaillement favorise l'adhésion des cellules à la matrice extracellulaire et contribue ainsi au maintien de la structure de l'épithélium tubulaire. Cependant, une autre interprétation - opposée - peut être donnée. Dans la mesure où la formation de ces dômes résulte d'un transport vectoriel de fluide, elle suggère que les protéines de transport impliquées sont correctement réparties entre les domaines apicaux et basolatéraux des cellules MDCK, régionalisation qui signe la mise en place d'une polarité cellulaire. La disparition des dômes en réponse au shear stress signifierait alors que le FSS urinaire altère la polarité des cellules tubulaires.
Quid de la détection du cisaillement?

Dans le tubule proximal, ce sont les microvillosités apicales qui seraient les senseurs précoces du FSS. Les microvillosités sont des projections en doigts de gant de la membrane apicale qui renferment un faisceau de filaments d'actine orientés selon l'axe apical/basal. À leur base, les microfilaments adhèrent à un second réseau d'actine, organisé sous la membrane plasmique apicale. Les modèles proposés par Guo et al. [18] et le groupe de Wang $[14,15]$ indiquent que sous l'influence du FSS urinaire, les microvillosités se courbent et les filaments d'actine qu'elles contiennent se déforment. Les forces de torsion ainsi générées sont transmises au réseau d'actine sous-membranaire, 


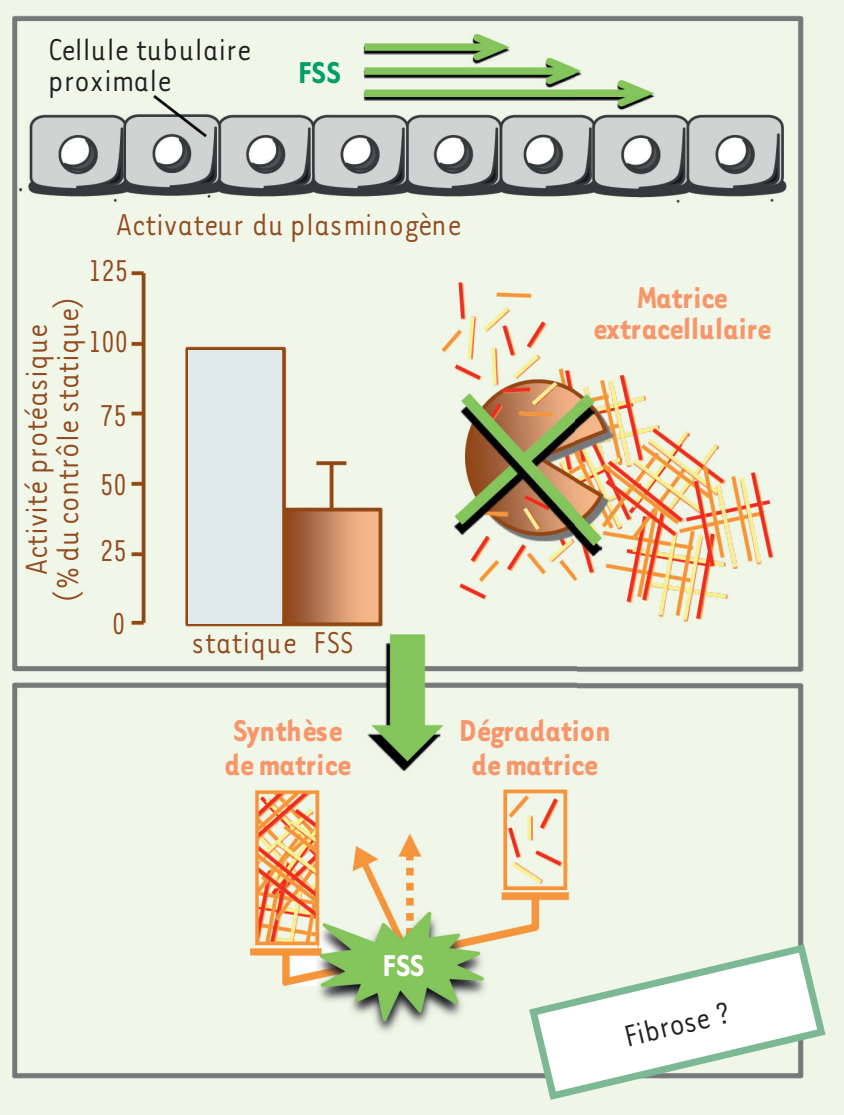

Figure 2. Le cisaillement urinaire inhibe les activateurs du plasminogène. L'exposition des cellules tubulaires proximales à un FSS entraîne une inhibition des deux activateurs du plasminogène, tPA (tissue-type plasminogen activator) et uPA (urokinase) [12]. Dans la mesure où ces protéases sont capables de dégrader les protéines matricielles, cela suggère que le FSS pourrait favoriser l'accumulation de matrice extracellulaire - ou fibrose - observée dans la majorité des pathologies rénales. C'est cette étude [12] qui a suggéré la première que le cisaillement urinaire jouerait potentiellement un rôle important dans la progression des néphropathies. FSS : fluid shear stress.

lequel déclenche une signalisation intracellulaire [19]. Dans le tubule distal et le canal collecteur en revanche, c'est plutôt le cil primaire qui servirait d'antenne pour le FSS. Le cil primaire est une excroissance de la membrane plasmique apicale, organisée autour des microtubules et présente dans vraisemblablement toutes les cellules épithéliales, excepté les cellules intercalaires du canal collecteur. C'est la courbure du cil primaire qui constituerait le premier évènement induit par le shear stress [20]. Elle provoquerait ensuite la réponse cellulaire grâce à deux mécanismes: la torsion des microtubules du cil, qui se propage aux microfilaments de la base du cil, ou l'étirement de la membrane ciliaire, qui induit une entrée de calcium via l'ouverture de canaux calciques sensibles à l'étirement $[19,21]$. Compte tenu de ces mécanismes, on comprend assez aisément que la hauteur/ densité des microvillosités et la longueur du cil primaire jouent un rôle important dans la réponse cellulaire au shear stress.
D'autres candidats pour la perception du cisaillement urinaire ont été évoqués. II s'agit par exemple du glycocalyx, revêtement cellulaire riche en glycoprotéines récemment impliqué dans la détection du FSS vasculaire, ou encore du canal sodique ENac $[1,10]$.

\section{Perturbation du cisaillement urinaire : un nouvel acteur des maladies rénales?}

\section{Des néphropathies associées à une modification du cisaillement}

L'analyse des pathologies rénales chez l'homme révèle qu'il existe probablement, dans la majorité d'entre elles, une modification du shear stress urinaire. Cette altération est la plupart du temps liée à des variations du débit du fluide urinaire. Dans les néphropathies obstructives par exemple, un obstacle gêne l'écoulement de l'urine, ce qui réduit le cisaillement urinaire. Dans les situations de réduction néphronique, la suppression d'une partie de la masse rénale entraîne une élévation compensatoire du débit de filtration glomérulaire dans les néphrons restants, à l'origine d'une augmentation du FSS. Au cours des transplantations rénales, la période ischémique s'accompagne d'une stagnation de l'urine dans la lumière tubulaire, responsable de l'abolition du FSS urinaire. En revanche, la phase de reperfusion entraîne une reprise de l'écoulement urinaire avec, par conséquent, une ré-induction du FSS. Enfin, dans les phases précoces des néphropathies diabétique ou hypertensive, un shear stress urinaire élevé s'opère secondairement à l'hyperfiltration glomérulaire. Dans d'autres cas, plus rares, l'altération du cisaillement urinaire résulte d'un changement de la viscosité de I'urine (hématurie ou protéinurie) ou du diamètre tubulaire (pathologies obstructives).

\section{Une perception du cisaillement abolie dans la polykystose}

La polykystose rénale autosomique dominante est une maladie héréditaire fréquente, caractérisée par la présence de nombreux kystes rénaux. Ces derniers se développent à partir de quelques tubules rénaux dont les cellules prolifèrent de manière excessive. Ces kystes adoptent une moindre différenciation et présentent des anomalies de polarité, responsables d'une hypersécrétion de fluide. L'augmentation du volume des kystes comprime progressivement les néphrons sains et entraîne leur destruction. Les gènes mutés en cause sont ceux qui codent pour les polycystines 1 et 2 . Principalement localisées dans la membrane du cil primaire, ces protéines forment un complexe moléculaire qui convertit la perception du FSS urinaire en une entrée 


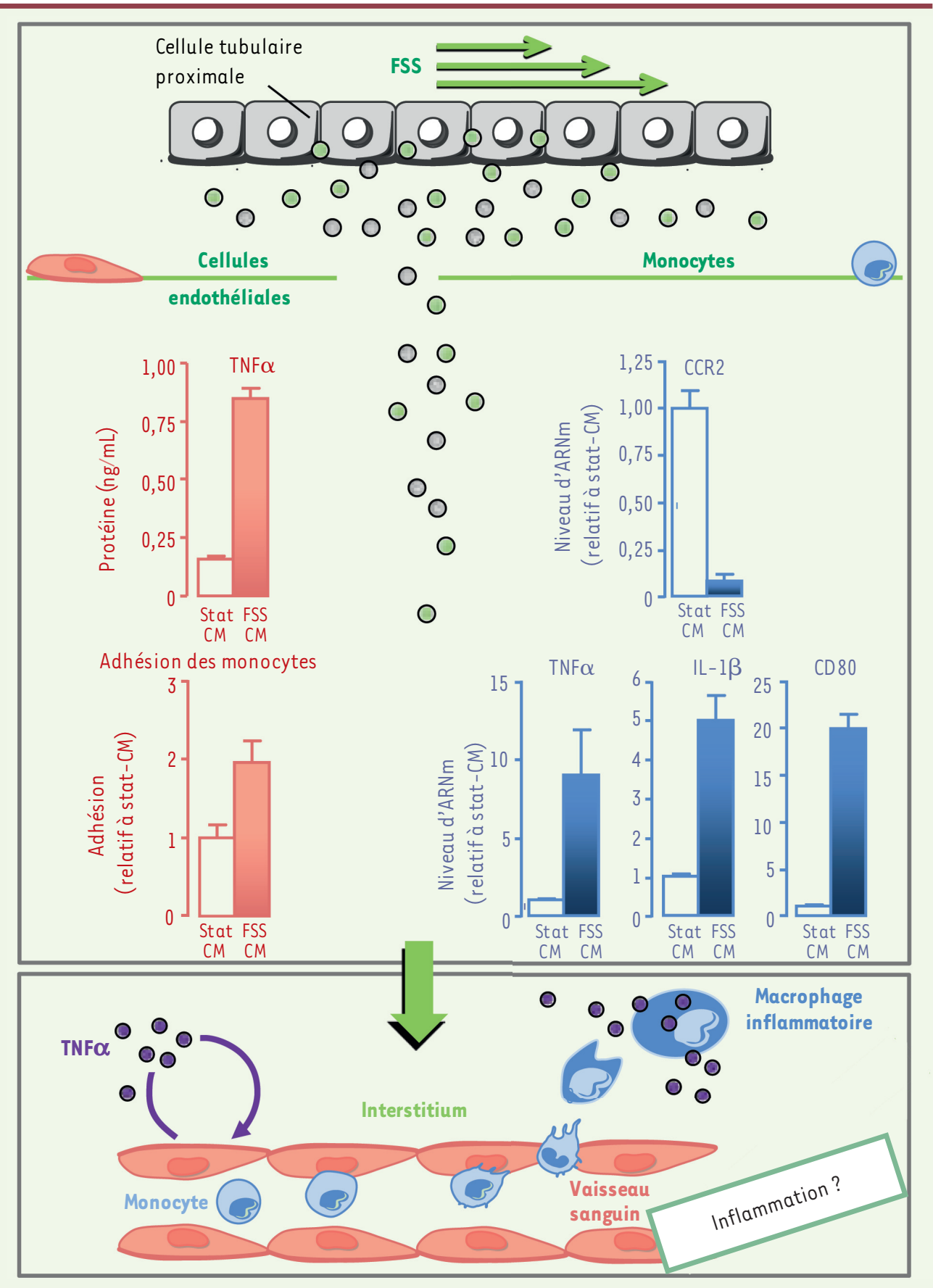

Figure 3. Le cisaillement urinaire : un facteur proinflammatoire. Lorsque des cellules tubulaires proximales sont sujettes à un FSS, elles libèrent de nombreuses molécules actives dans leur milieu environnant : ces molécules (FSS-CM, pour milieu conditionné par les cellules soumises au FSS) stimulent la libération par les cellules endothéliales de TNF $\alpha$ (tumor necrosis factor alpha) qui, à son tour, active l'adhésion des monocytes sur le tapis endothélial [3]. Le FSS-CM entraîne également un changement de phénotype des monocytes qui perdent leur marqueur CCR2 et acquièrent des marqueurs spécifiques des macrophages inflammatoires, tels que les cytokines TNF $\alpha$ et IL- $1 \beta$, ainsi que la protéine de surface CD80 [2]. Ainsi, le FSS urinaire pourrait promouvoir le recrutement des monocytes circulants dans l'interstitium, puis leur différenciation/activation en macrophages inflammatoires. Le cisaillement urinaire participerait donc au développement des néphropathies en facilitant l'inflammation. Stat-CM : milieu conditionné par les cellules non soumises au FSS (statique).

de calcium dans les cellules tubulaires [22]. Ainsi, la polykystose correspond à une altération des systèmes de détection du FSS et non du shear stress urinaire lui-même (au moins dans les phases initiales) et, pour les cellules, tout se passe comme-ci elles n'étaient plus exposées au FSS physiologique.

\section{Des protéines impliquées dans les néphropathies sont des cibles du cisaillement}

Des travaux effectués in vitro montrent que les cibles moléculaires du cisaillement sont des protéines qui participent au développement des maladies rénales. La majorité des études sur ce sujet ont été réalisées sur des cellules rénales représentatives du tubule proximal. Une étude ancienne indique que le FSS diminue l'expression de la superoxide dismutase [23]. Dans la mesure où cette protéine est une enzyme antioxydante, ce résultat suggère que le FSS pourrait promouvoir le stress oxydant observé dans les néphropathies. Cette même étude rapporte aussi que le FSS stimule l'expression de la cubiline et de la mégaline, deux protéines membranaires servant de récepteurs 
scavenger pour d'autres protéines. Or, la mégaline peut s'avérer délétère pour le tubule rénal. En effet, l'induction d'une protéinurie chez des souris mosaïques chez lesquelles $50 \%$ des cellules du tubule proximal rénal n'expriment pas la mégaline révèle que les cellules mégaline $^{+/+}$absorbent/accumulent davantage de protéines filtrées et expriment davantage de marqueurs de lésions tubulaires que les cellules mégaline ${ }^{-/-}$[24]. En stimulant la mégaline, le FSS pourrait alors contribuer à la toxicité des protéines anormalement filtrées.

La fibrose est une accumulation exagérée de matrice extracellulaire qui est présente dans la majorité des pathologies rénales. Son apparition dans l'espace interstitiel entre les tubules rénaux est fortement corrélée à la perte de la fonction rénale [25]. Il a été montré que le FSS réduit l'expression génique et protéique des activateurs du plasminogène uPA (urokinase) et $\mathrm{tPa}$ (tissue-type plasminogen activator) et inhibe leur activité [12] (Figure 2). Ces deux protéases participent directement ou indirectement à la dégradation des protéines matricielles. En les inhibant, le FSS urinaire pourrait alors induire un déséquilibre entre la synthèse et la dégradation de la matrice extracellulaire, contribuant ainsi au développement de la fibrose tubulointerstitielle [12]. Le FSS est aussi capable de modifier la localisation des récepteurs de type 1 de l'angiotensine-II (ATI). L'angiotensine-II est un acteur clé dans l'installation de la fibrose rénale [25] et les seuls médicaments actuellement utilisés chez l'homme pour ralentir la fibrogenèse sont ceux qui bloquent l'action de l'angiotensine-II (inhibiteurs de l'enzyme de conversion et antagonistes des récepteurs AT1). Le FSS induit l'externalisation à la membrane plasmique apicale des récepteurs $A T l$, ceux-ci étant concentrés dans des endosomes subapicaux proches du cil primaire en conditions statiques [26]. En provoquant cette relocalisation, le FSS pourrait donc potentialiser la réponse du tubule rénal à l'angiotensine-II.

Enfin, dans les cellules issues de canal collecteur, le FSS augmente l'expression de l'endothéline [27]. Sachant que les antagonistes des récepteurs à l'endothéline ralentissent la progression des lésions rénales dans les modèles animaux de néphropathies [28, 29], cela signifie que le FSS pourrait participer à la destruction des néphrons par le biais de l'endothéline.

Tous ces résultats suggèrent donc que le cisaillement urinaire constitue un facteur d'agression pour le tubule rénal. II vient d'ailleurs d'être montré que l'exposition de cellules tubulaires humaines (cellules HK-2) à un FSS entraîne la production de KIMl (kidney injury molecule 1) et NGAL (neutrophil gelatinase-associated lipocalin), des marqueurs de lésions tubulaires [2].

\section{Rôle du cisaillement dans l'inflammation rénale}

L'inflammation est une étape clé et précoce dans la progression des néphropathies. Elle précède l'accumulation de matrice extracellulaire et se déclenche sous l'influence des médiateurs inflammatoires que libèrent les cellules tubulaires agressées [25]. Deux études récentes ont montré que le FSS urinaire pourrait promouvoir l'inflammation. Premièrement, le traitement de cellules endothéliales avec du milieu conditionné par des cellules tubulaires soumises à un cisaillement entraîne la sécrétion de la cytokine inflammatoire TNF $\alpha$ (tumor necrosis factor $\alpha$ ) [3]. Puis, par un mécanisme autocrine ou paracrine, ce TNF $\alpha$ stimule la sécrétion de la chimiokine CCL2 et l'expression de la protéine d'adhésion VCAM-1 (vascular cell adhesion molecule-1), permettant ainsi l'adhésion des monocytes [3] (Figure 3). Par le biais de cette activation endothéliale, le FSS tubulaire faciliterait donc le recrutement des monocytes circulants dans l'interstitium tubulaire, les monocytes représentant la population de leucocytes infiltrés la plus importante et la plus fortement impliquée dans l'installation de la fibrose [25]. Deuxièmement, le surnageant des cellules cisaillées mis en présence de monocytes inhibe dans ces cellules l'expression du marqueur monocytaire CCR2 [2]. En revanche, il stimule celle des marqueurs macrophagiques inflammatoires, tels que le TNF $\alpha$, I'interleukine-1 $\beta$ (IL-1 $\beta$ ) et CD80, et augmente la sécrétion de TNF $\alpha$ [2] (Figure 3). En réponse au FSS tubulaire, les monocytes humains se différencieraient donc en macrophages inflammatoires. Délétères in vivo, ces macrophages contribuent à l'extension des lésions et à la progression de la fibrose en produisant des radicaux libres oxygénés et en sécrétant des cytokines proinflammatoires, toxiques pour les cellules résidentes rénales [25].

\section{Conclusion}

L'écoulement de l'urine et le cisaillement qu'il provoque sur la paroi du tubule rénal ne sont donc probablement pas anodins pour le rein. La réponse des cellules tubulaires aux variations de cisaillement semble être importante pour l'ajustement de la fonction rénale aux besoins de l'organisme. Lorsque cette réponse est diminuée, ou lorsque les variations du FSS urinaire deviennent chroniques, des anomalies peuvent apparaître, ce qui conduit au développement des maladies rénales. Les agressions initiales des cellules du tubule rénal, comme l'étirement en cas d'obstruction tubulaire, la présence de protéines anormalement filtrées dans les pathologies glomérulaires, ou l'environnement riche en glucose dans le diabète, constituent des événements clés dans l'installation de la fibrose et la progression des néphropathies [25]. Au vu des travaux in vitro portant sur le FSS, il convient maintenant d'ajouter à cette liste le cisaillement urinaire.

Les recherches futures devront déterminer les mécanismes moléculaires qui convertissent le FSS urinaire en une réponse biologique. La compréhension de ces mécanismes pourrait déboucher sur de nouvelles stratégies thérapeutiques. En effet, la découverte de substances modulant les systèmes de perception/transduction du FSS urinaire devrait permettre de ralentir la 
progression de l'insuffisance rénale en libérant les cellules rénales des contraintes liées aux variations du flux urinaire. $\diamond$

\section{SUMMARY}

Renal urinary shear stress: a novel actor in nephropathies

The role of fluid shear stress is well established in vascular pathophysiology. However, urinary shear stress now also appears as a key mechanism in the regulation of renal function. In addition, there is a growing body of evidence showing that modified urinary shear stress is involved in the development of nephropathies. Therefore we review here the state-of-the-art on the pathophysiological roles of urinary shear stress. $\diamond$

\section{LIENS D'INTÉRÊT}

Les auteurs déclarent n'avoir aucun lien d'intérêt concernant les données publiées dans cet article.

\section{REMERCIEMENTS}

Bénédicte Buffin-Meyer remercie l'association 111 des Arts pour son soutien.

\section{RÉFÉRENCES}

1. Ando J, Yamamoto K. Effects of shear stress and stretch on endothelial function. Antioxid Redox Signal $2011 ; 15: 1389-403$.

2. Miravète $M$, Dissard R, Klein J, et al. Renal tubular fluid shear stress facilitates monocyte activation towards inflammatory macrophages. Am J Physiol Renal Physiol 2012 ; 302 : F1409-17.

3. Miravète $M$, Klein J, Besse-Patin A, et al. Renal tubular fluid shear stress promotes endothelial cell activation. Biochem Biophys Res Commun $2011 ; 407: 813-7$.

4. Duan $Y$, Weinstein $A M$, Weinbaum $S$, et al. Shear stress-induced changes of membrane transporte localization and expression in mouse proximal tubule cells. Proc Natl Acad Sci USA $2010 ; 107$ : 21860-5.

5. Jang KJ, Cho HS, Kang Do H, et al. Fluid-shear-stress-induced translocation of aquaporin-2 and reorganization of actin cytoskeleton in renal tubular epithelial cells. Integr Biol (Camb) 2011 ; 3 : 134-41.

6. Holtzclaw JD, Liu L, Grimm PR, et al. Shear stress-induced volume decrease in C11-MDCK cells by BK-alpha/beta4. Am J Physiol Renal Physiol 2010 ; 299 : F507-16.

7. Cabral PD, Hong NJ, Garvin JL. Shear stress increases nitric oxide production in thick ascending limbs. Am J Physiol Renal Physiol 2010 ; 299 : Fl185-92.

8. Cai Z, Xin J, Pollock DM, et al. Shear stress-mediated NO production in inner medullary collecting duct cells. Am J Physiol Renal Physiol $2000 ; 279$ : F270-4.

9. Flores $\mathrm{D}$, Liu Y, Liu W et al. Flow induced prostaglandin $\varepsilon 2$ release regulates $\mathrm{Na}$ and $\mathrm{K}$ transport in the collecting duct. Am J Physiol Renal Physiol 2012 ; 303 : F632-8.

10. Carattino MD, Sheng S, Kleyman TR. Epithelial $\mathrm{Na}+$ channels are activated by laminar shear stress. J Biol Chem $2004 ; 279: 4120-6$.

11. Duan Y, Gotoh N, Yan Q, et al. Shear-induced reorganization of renal proximal tubule cell actin cytoskeleton and apical junctional complexes. Proc Natl Acad Sci USA 2008 ; 105 : 11418-23.

12. Essig M, Terzi F, Burtin M, et al. Mechanical strains induced by tubular flow affect the phenotype of proximal tubular cells. Am J Physiol Renal Physiol 2001 ; 281 : F751-62.

13. Essig $M$, Friedlander $G$. Tubular shear stress and phenotype of renal proximal tubular cells. J Am Soc Nephrol 2003 ; 14 : S33-5.
14. Du Z, Yan Q, Duan Y, et al. Axial flow modulates proximal tubule NHE3 and $\mathrm{H}$-ATPase activities by changing microvillus bending moments. Am J Physiol Renal Physiol 2006 ; 290 : F289-96.

15. Du Z, Duan Y, Yan $Q$, et al. Mechanosensory function of microvilli of the kidney proximal tubule. Proc Natl Acad Sci USA 2004 ; 101 : 13068-73.

16. Alenghat FJ, Nauli SM, Kolb R, et al. Global cytoskeletal control of mechanotransduction in kidney epithelial cells. Exp Cell Res 2004 ; 301 : $23-30$.

17. Cattaneo I, Condorelli L, Terrinoni AR, et al. Shear stress reverses dome formation in confluent renal tubular cells. Cell Physiol Biochem $2011 ; 28$ : 673-82.

18. Guo $P$, Weinstein AM, Weinbaum $S$. A hydrodynamic mechanosensory hypothesis for brush border microvilli. Am J Physiol Renal Physiol $2000 ; 279$ : F698-712.

19. Weinbaum S, Duan Y, Satlin LM, et al. Mechanotransduction in the renal tubule. Am J Physiol Renal Physiol 2010 ; 299 : F1220-36.

20. Praetorius HA, Spring KR. The renal cell primary cilium functions as a flow sensor. Curr Opin Nephrol Hypertens 2003 ; 12 : 517-20.

21. Liu W, Xu S, Woda C, et al. Effect of flow and stretch on the $\left[\mathrm{Ca}^{2+}\right]$ i response of principal and intercalated cells in cortical collecting duct. Am J Physiol Renal Physiol 2003 ; 285 : F998-1012.

22. Nauli SM, Alenghat FJ, Luo Y, et al. Polycystins 1 and 2 mediate mechanosensation in the primary cilium of kidney cells. Nat Genet 2003 ; $33: 129-37$.

23. Kaysen JH, Campbell WC, Majewski RR, et al. Select de novo gene and protein expression during renal epithelial cell culture in rotating wall vessels is shear stress dependent. J Membr Biol $1999 ; 168: 77-89$.

24. Motoyoshi Y, Matsusaka T, Saito A et al. Megalin contributes to the early injury of proximal tubule cells during nonselective proteinuria. Kidney Int $2008 ; 74: 1262-9$

25. Klein J, Miravete M, Buffin-Meyer B, et al. La fibrose tubulo-interstitielle rénale-Menace fantôme ou dernière croisade ? Med Sci (Paris) 2011 ; 27 : 55-61

26. Kolb RJ, Woost PG, Hopfer U. Membrane trafficking of angiotensin receptor type- 1 and mechanochemical signal transduction in proximal tubule cells. Hypertension $2004 ; 44: 352-9$.

27. Lyon-Roberts $B$, Strait KA, van Peursem $\varepsilon$, et al. Flow regulation of collecting duct endothelin-1 production. Am J Physiol Renal Physiol 2011 ; 300 : F650-6.

28. Boffa JJ, Dussaule JC, Ronco $P$, et al. Maladie rénale chronique, les voies de recherche thérapeutique. Rev Prat $2012 ; 62: 72-5$

29. Kohan DE, Pritchett $Y$, Molitch M, et al. Addition of atrasentan to reninangiotensin system blockade reduces albuminuria in diabetic nephropathy. $J$ Am Soc Nephrol 2011 ; 22 : 763-72.

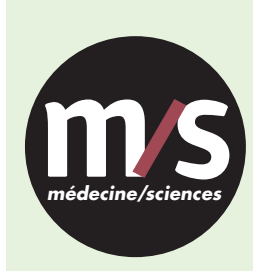

Tarifs d'abonnement $\mathrm{m} / \mathrm{s}-2013$

Abonnez-vous

à médecine/sciences
$>$ Grâce à $m / s$, vivez en direct les progrès des sciences biologiques et médicales

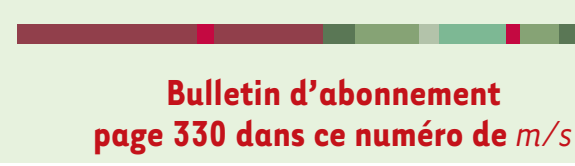

TIRÉS À PART

B. Buffin-Meyer 